\title{
Abundances of planetary nebula M 1-42 ${ }^{\star}$
}

\author{
S. R. Pottasch ${ }^{1}$, J. Bernard-Salas ${ }^{2}$, and T. L. Roellig ${ }^{3}$ \\ 1 Kapteyn Astronomical Institute, PO Box 800, 9700 AV Groningen, The Netherlands \\ e-mail: pottasch@astro.rug.nl \\ 2 Center for Radiophysics and Space Research, Cornell University, Ithaca, NY 14853, USA \\ 3 NASA Ames Research Center, MS 245-6, Moffett Field, CA 94035-1000, USA
}

Received 1 March 2007 / Accepted 19 June 2007

ABSTRACT

\begin{abstract}
The spectra of the planetary nebula M 1-42 is reanalysed using spectral measurements made in the mid-infrared with the Spitzer Space Telescope. The aim is to determine the chemical composition of this object. We also make use of ISO, IUE and ground based spectra. Abundances determined from the mid- and far-infrared lines, which are insensitive to electron temperature, are used as the basis for the determination of the composition, which are found to substantially differ from earlier results. High values of neon, argon and sulfur are found. They are higher than in other PN, with the exception of NGC 6153, a nebula of very similar abundances. The high values of helium and nitrogen found indicate that the second dredge-up and hot bottom burning has occurred in the course of evolution and that the central star was originally more massive than $4 M_{\odot}$. The present temperature and luminosity of the central star is determined and at first sight may be inconsistent with such a high mass.
\end{abstract}

Key words. ISM: abundances - planetary nebulae: individual: M 1-42 - infrared: ISM

\section{Introduction}

M 1-42 (PN G2.7-04.8) is a rather faint planetary nebula (PN) with a high radial velocity which is located in the direction of the galactic center. For this reason it is considered by Acker et al. (1992) to be a galactic bulge PN. It has been studied earlier by Liu et al. (2001) who found that it has a very low Balmer jump temperature and the abundances found from the measured recombination lines are an order of magnitude higher than those found from the collisional excited lines.

The purpose of this paper is to study the element abundances in this nebula with the help of mid-infrared spectra taken by the Spitzer Space Telescope (Werner et al. 2004). Abundances in this nebula have been studied earlier by Exter et al. (2004), Aller \& Keyes (1979) and Ratag et al. (1997) using only the visual spectrum as well as by Liu et al. (2001) who also included ISO measurements. The use of the mid- and far-infrared spectrum permits a more accurate determination of the abundances for reasons that have been discussed in earlier papers (e.g. see Pottasch \& Beintema 1999; Pottasch et al. 2000, 2001; Bernard Salas et al. 2001), and can be summarized as follows.

The most important advantage is that the infrared lines originate from very low energy levels and thus give an abundance which is not sensitive to the temperature in the nebula, nor to possible temperature fluctuations. Furthermore, when a line originating from a high-lying energy level in the same ion is observed, it is possible to determine an effective (Boltzmann) temperature at which the lines in that particular ion are formed. When the effective temperature for many ions can be determined, it may be possible to make a plot of effective temperature against ionization potential, which can be used to determine the

^ Based on observations with the Spitzer Space Telescope, which is operated by the Jet Propulsion Laboratory, California Institute of Technology. effective temperature for ions for which only lines originating from a high energy level are observed. This may not work if the same ion is present in a range of electron temperatures.

Use of the infrared spectra have further advantages. One of them is that the number of observed ions used in the abundance analysis is approximately doubled, which removes the need for using large "Ionization Correction Factors" (ICFs), thus substantially lowering the uncertainty in the abundance. A further advantage is that the extinction in the infrared is almost negligible, eliminating the need to include sometimes large correction factors. This is especially important when the extinction is uncertain. As mentioned above, Liu et al. (2001) used the ISO midand far-infrared observations in their analysis. We have used the IRS spectrograph of the Spitzer Space Telescope to observe M1-42 in the same spectral region as the ISO SWS measurements. We report different results, which are probably due to two circumstances. First of all the SWS measurement was not well centered on the nebula. Together with the fact that the nebula is rather large (about $11^{\prime \prime}$ in diameter), this means that part of the nebula may be missed in the ISO measurement (this is also true of the IRS short wavelength slit). Furthermore the observed spectrum has low intensity so that the errors in the ISO SWS intensities are high.

This paper is structured as follows. First the IRS spectrum of M1-42 is presented and discussed (in Sect. 2). Then the intrinsic $\mathrm{H} \beta$ flux is determined using both the measurements of the infrared hydrogen lines and the radio continuum flux density (Sect. 3). The visible spectrum of the nebula is presented in Sect. 4 together with a new reduction of the ultraviolet (IUE) spectrum. This is followed by a discussion of the nebular electron temperature and density and the chemical composition (Sect. 4). A comparison of the resultant abundances with those made earlier is given in Sect. 5. In this section the relation between nebular abundances determined from collisionally excited 


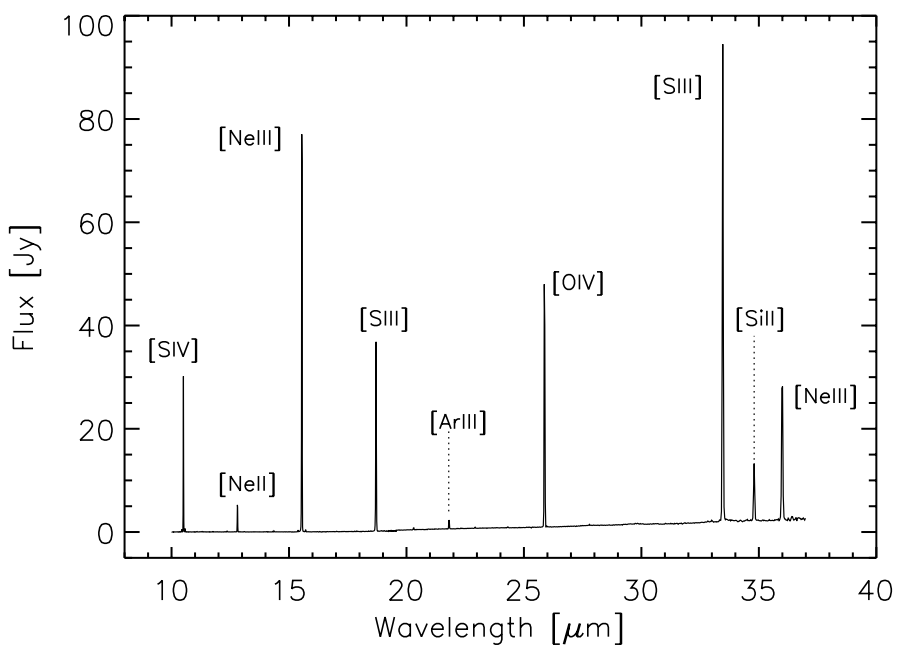

Fig. 1. Observed Spitzer-IRS high-resolution spectrum of M 1-42. The most prominent lines are labeled in the figure.

lines and from recombination lines is also discussed, as well as the striking similarity of the abundances obtained with those of NGC 6153. In Sect. 6 discussion and concluding remarks are given.

\section{The Infrared spectra}

\subsection{IRS observations of M 1-42}

The IRS spectrograph (Houck et al.2004) on board the Spitzer Space Telescope is similar to the ISO SWS spectrometer, but has higher sensitivity. The IRS high resolution spectra have a spectral resolution $(\lambda / \delta \lambda)$ of about 600 which is less than the ISO SWS by at least a factor of two. The aperture size is comparable for the two instruments. The SH has a slit size of $4.7^{\prime \prime} \times 11.3^{\prime \prime}$, while the LH is $11.1^{\prime \prime} \times 22.3^{\prime \prime}$, the latter being close to the smaller SWS aperture. The IRS high resolution measures in two spectral ranges: the short high (SH) going from $9.9 \mu \mathrm{m}$ to $19.6 \mu \mathrm{m}$ and the long high (LH) from $18.7 \mu \mathrm{m}$ to $37.2 \mu \mathrm{m}$. The observed IRS spectrum of M 1-42 is shown in Fig. 1. Besides the usual strong lines of neon, sulfur, oxygen and argon, many other lines have also been measured in the spectrum, including molecular hydrogen. The ISO SWS has about the same upper wavelength but extends on the short wavelength end to about $2.5 \mu \mathrm{m}$.

The IRS measurement of M1-42 was centered at RA(2000) $18^{\mathrm{h}} 11^{\mathrm{m}} 04.87^{\mathrm{s}}$ and $\operatorname{Dec}(2000)-28^{\circ} 58^{\prime} 59.4^{\prime \prime}$. This may be compared with the value measured by Kerber et al. (2003) of $\operatorname{RA}(2000) 18^{\mathrm{h}} 11^{\mathrm{m}} 05.028^{\mathrm{s}}$ and $\operatorname{Dec}(2000)-28^{\circ} 58^{\prime} 59.33^{\prime \prime}$ from visual measurements. The ISO measurement was made at $\mathrm{RA}(2000) 18^{\mathrm{h}} 11^{\mathrm{m}} 04.6^{\mathrm{s}}$ and $\operatorname{Dec}(2000)-28^{\circ} 59^{\prime} 00.6^{\prime \prime}$. which is about $6.5^{\prime \prime}$ from the position given by Kerber et al. (2003) A comparison of a photograph of the nebula with the apertures superimposed indicates that the LH aperture measured the entire nebula, while the SH aperture missed part of the nebula. A correction was made for the missing emission by making use of the fact that the two spectrographs had a wavelength region in common at about $19 \mu \mathrm{m}$. To make the continuum emission at this wavelength equal, the $\mathrm{SH}$ emission had to be increased by a factor of 1.8. This factor is approximately the ratio of the total area of the nebula to the area measured measured in the $\mathrm{SH}$ slit $\left(4.7 \times 11.3^{\prime \prime}\right)$. This indicates that the emission from the nebula is homogeneous. The measured emission line intensities are given in Table 1, where the actual measurements are given in the
Table 1. The measured lines are given in Col. 3. The last column gives the ratio of the line intensity to $\mathrm{H} \beta(=100)$.

\begin{tabular}{|c|c|c|c|}
\hline Ident. & $\lambda(\mu \mathrm{m})$ & Intensity $^{\dagger}$ & $\overline{\mathrm{I} / \mathrm{H} \beta^{\sharp}}$ \\
\hline & 9.98 & 6.52 & 0.96 \\
\hline [S IV] & 10.51 & 1665 & 321 \\
\hline Н I 9-7 & 11.30 & 4.27 & 0.806 \\
\hline [Cl IV] & 11.76 & 4.50 & 0.844 \\
\hline $\mathrm{H}_{2}$ & 12.28 & 3.53 & 0.65 \\
\hline H I7-6(11-8) & 12.37 & 10.2 & 1.89 \\
\hline$[\mathrm{Ne} \mathrm{II}]$ & 12.81 & 235 & 43.5 \\
\hline [Ar V] & 13.10 & 3.41 & 0.63 \\
\hline$[\mathrm{Ne} \mathrm{V}]$ & 14.31 & 2.78 & 0.51 \\
\hline [Cl II] & 14.36 & 7.68 & 1.41 \\
\hline [Ne III] & 15.55 & 2708 & 497 \\
\hline $\mathrm{H}_{2}$ & 17.03 & 1.83 & 0.336 \\
\hline [S III] & 18.70 & 994 & 184 \\
\hline [Cl IV] & 20.31 & 10.47 & 1.07 \\
\hline [Ar III] & 21.81 & 41.0 & 4.19 \\
\hline [Fe III $]$ & 22.92 & 3.68 & 0.376 \\
\hline$[\mathrm{Ne} \mathrm{V}]$ & 24.31 & 3.89 & 0.396 \\
\hline [O IV] & 25.87 & 900 & 91.5 \\
\hline [Fe II] & 25.98 & 2.51 & 0.255 \\
\hline H I9-8 & 27.78 & 2.66 & 0.27 \\
\hline [S III] & 33.46 & 1185 & 120 \\
\hline [Si II] & 34.80 & 158 & 16.0 \\
\hline [Ne III] & 35.99 & 424 & 42.8 \\
\hline
\end{tabular}

The intensity is measured in units of $10^{-14} \mathrm{erg} \mathrm{cm}^{-2} \mathrm{~s}^{-1}$.

$\sharp$ In the ratio of intensity to $\mathrm{H} \beta$ the intensities below $19 \mu \mathrm{m}$ are multiplied by a factor 1.8 to correct for part of the nebula being outside the diaphragm. A reddening correction is also made but it is very small.

column labeled "intensity". In the column giving the ratio of the intensity to $\mathrm{H} \beta$ the $\mathrm{SH}$ intensity includes the correction factor of 1.8; it also includes a small correction for extinction using the extinction value given below and the extinction curve given by Fluks et al. (1994). The $\mathrm{H} \beta$ intensity is discussed in Sect. 3.

There are other ways of determining the factor which accounts for the emission missing in the SH aperture. The method given above, that of making use of the fact that the continua in the region of wavelength overlap should be equal, is theoretically satisfying. But any pair of lines can also be used as long as they originate from the same ion and two other conditions exist: that each of the lines is measured in a different aperture, and that the theoretical value of the ratio of the two lines is only weakly dependent on the electron temperature and density. Five candidate pairs of lines exist. The best is the ratio of the [Ne III] lines at $\lambda 15.5$ and $\lambda 36.0 \mu \mathrm{m}$. Theoretically the ratio of the intensities of these lines should be about 11.8 , with only a small dependance on nebular temperature and density. Our measurements of these line pairs again leads to a value of 1.8 , the same correcting factor given as by the continua. The [ $\mathrm{Ne} \mathrm{V}]$ and [Cl IV] line pairs are only slightly more dependant on the nebular properties and they are consistent with the value of 1.8. Only the hydrogen lines do not give consistent values. A value of 1.5 is obtained from the $\lambda 12.38 \mu \mathrm{m}$ line, if it is a blend of the $\lambda 12.372 \mu \mathrm{m}$ and the $\lambda 12.387 \mu \mathrm{m} 7-6$ and $11-8$ transitions of hydrogen. While this could be an error in the intensity measured for this line, it is more likely there is another line which contributes to the measured intensity. The He I (7-6) transition is a likely possibility. The line at $\lambda 11.30 \mu \mathrm{m}$ also appears to be a blend of the hydrogen line with a helium line and/or a line of [Cl I]. It thus appears that the total emission in the SH wavelength band is obtained by increasing the measured intensities by a factor of $1.8 \pm 0.2$. 
Table 2. ISO LWS spectrum of M 1-42. The measured lines are given in Col. 3 . The last column gives the ratio of the line intensity to $\mathrm{H} \beta(=100)$. The intensities are measured in units of $10^{-14} \mathrm{erg} \mathrm{cm}^{-2} \mathrm{~s}^{-1}$. No reddening correction has been made.

\begin{tabular}{lccc}
\hline \hline Ident. & $\lambda(\mu \mathrm{m})$ & Intensity & $\mathrm{I} / \mathrm{H} \beta$ \\
\hline$[\mathrm{O} \mathrm{III}]$ & 51.83 & 3830 & 383 \\
{$[\mathrm{~N} \mathrm{III}]$} & 57.32 & 2690 & 269 \\
{$[\mathrm{O} \mathrm{I}]$} & 63.19 & 318 & 31.8 \\
{$[\mathrm{O}$ III $]$} & 88.35 & 1800 & 180 \\
{$[\mathrm{~N} \mathrm{II}]$} & 121.9 & 59 & 5.9 \\
\hline
\end{tabular}

\subsection{Comparison with the ISO SWS spectrum}

Comparing the present intensities with those found by ISO SWS (de Graauw et al. 1999) measurements given by Liu et al. (2001) is complicated by the mispointing of the ISO measurement. In addition, the ISO SWS observation of M 1-42 is noisy and is compromised by an inconsistency between the up and down scans. The up scan shows no line emission (the spectrum seems pure noise) while the down scan does have line emission. The strongest line in the SWS spectrum (S III line at $18.7 \mu \mathrm{m}$ ) in the down scan shows a strange blue wing which is completely absent in the IRS spectrum. The nature of these problems is not known (both scans should show the same spectrum) and makes the SWS data of this object suspicious. The two lines measured by ISO SWS with the largest aperture (above $29 \mu \mathrm{m}$ the ISO aperture was $20^{\prime \prime} \times 33^{\prime \prime}$ ) have intensities within $20 \%$ of the values we have found, which, considering the rather low quality of these ISO measurements, is reasonable agreement. The [O IV] at $25.9 \mu \mathrm{m}$ measured by ISO is more than a factor of 3 smaller than our value. At least part of this difference is probably because the ISO aperture is now smaller than for the two longer wavelength lines. The three ISO lines measured shortward of $19 \mu \mathrm{m}$ ([S IV], [Ne III] and [S III]) are about 30-40\% less intense than Spitzer has measured. Because the Spitzer measurements have to be increased by a factor of about 1.8, the ISO measurements made with the $14^{\prime \prime} \times 27^{\prime \prime}$ aperture of these three lines must be increased by about a factor of 3 .

\subsection{ISO LWS spectrum}

The ISO LWS (Clegg et al. 1996) observation of M 1-42 is used here because it is made with a much larger aperture which has a diameter of about $80^{\prime \prime}$ and includes the entire nebula. The on and off source spectra are designated TDT 70302104 and 70302105 respectively. Background emission is only important in the [N II] and [C II] lines: the latter line is unusable for this reason. The measured line intensities are given in Table 2.

\section{Extinction}

There are several methods for obtaining the extinction: (1) comparison of the radio emission with the $\mathrm{H} \beta$ flux; (2) comparison of the observed and theoretical Balmer decrement; (3) the dip at $\lambda 2200 \AA$ and; (4) photometry of the exciting star. Only the first two methods are applicable here.

\subsection{The $6 \mathrm{~cm}$ radio emission}

The $6 \mathrm{~cm}$ flux density has been measured with the VLA by Zijlstra et al. (1989). They find a value of $24 \mathrm{mJy}$. The nebula has also been measured at $21 \mathrm{~cm}$ by Condon \& Kaplan (1998) who find $28.6 \mathrm{mJy}$, which, if optically thin, would imply $24.2 \mathrm{mJy}$ at $6 \mathrm{~cm}$. Using values of $T_{\mathrm{e}}$ and helium abundance determined below together with the equation quoted in Pottasch (1984) this implies an $\mathrm{H} \beta$ flux of $8.4 \times 10^{-12} \mathrm{erg} \mathrm{cm}^{-2} \mathrm{~s}^{-1}$.

\subsection{The $H \beta$ flux}

The $\mathrm{H} \beta$ flux may also be found from the measured $\mathrm{HI}(9-8)$ transition at $27.80 \mu \mathrm{m}$ given in Table 1 . Using the theoretical value of the ratio of this transition to $\mathrm{H} \beta$ given by Hummer and Storey (1987) at $T_{\mathrm{e}}=7500 \mathrm{~K}$ and a density of $10^{3} \mathrm{~cm}^{-3}$

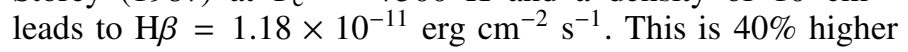
than the value found from the radio flux density. A value of $1.0 \times 10^{-11} \mathrm{erg} \mathrm{cm}^{-2} \mathrm{~s}^{-1}$ will be used in the remainder of the paper. The uncertainty is about $20 \%$. The HI(7-6) transition could also have been used to determine the $\mathrm{H} \beta$ flux. There are two reasons for not using this transition. Firstly it is in the spectral region where the slit did not cover the entire nebula. Secondly the line is blended with another hydrogen line $(\mathrm{HI}(11-8))$ and a helium line. Although approximately the same $\mathrm{H} \beta$ flux is obtained from this line, the uncertainty is somewhat larger.

\subsection{The Balmer decrement extinction $E_{B-V}$}

The extinction determined from the Balmer decrement differs somewhat according to the author. Liu et al. (2001) give $C=$ 0.71 , Aller \& Keyes (1979) give $C=0.60$, Exter et al. (2004) find $C=0.53$.

The measured value of the $\mathrm{H} \beta$ flux (which is affected by extinction) is $2.3 \times 10^{-12} \mathrm{erg} \mathrm{cm}^{-2} \mathrm{~s}^{-1}$ (Webster 1983) which leads to a value of $C=0.63$ or $E_{B-V}=0.43$. This is in reasonably good agreement with the values found from the Balmer decrement and will be used when necessary in this paper.

\subsection{The visual spectrum}

The visual spectrum has been measured by several authors. The highest resolution spectrum is by Liu et al. (2001), and reliable spectra have also been reported by Exter et al. (2004) and Aller $\&$ Keyes (1979). The measurements were made with slits which cover a reasonably large area of the nebula and may be considered as representative of the total nebula. The measurements of Liu et al. (2001) are preferred because of the higher spectral resolution used. Some weight is also given to the careful work of Exter et al. (2004). The results are shown in Table 3 where the Cols. 3-5 give the intensities measured by the various authors relative to $\mathrm{H} \beta=100$ for those lines which are of interest. The line intensities have been corrected by the individual authors for extinction. No attempt has been made to use a common extinction correction because all give a correct Balmer decrement. In the last column average values are given, with greater weight given to the measurements of the first two authors.

\subsection{The IUE ultraviolet spectrum}

Only a single low resolution IUE observation of this nebula exists. It was taken with the large aperature $\left(10^{\prime \prime} \times 23^{\prime \prime}\right)$ with an exposure time of $17100 \mathrm{~s}$. In spite of this long exposure time the spectrum is underexposed and only a few lines can be identified. Liu et al. (2001) have discussed this measurement and identified four lines in the spectrum. We have found the same four lines in our analysis. The line intensities have been rederived for us by W. A. Feibelman and differ somewhat from those given by 
Table 3. Visual spectrum of M 1-42.

\begin{tabular}{|c|c|c|c|c|c|}
\hline \multirow{2}{*}{$\begin{array}{c}\lambda \\
(\AA)\end{array}$} & \multirow[t]{2}{*}{ Ion } & \multicolumn{3}{|c|}{ Intensities } & Total \\
\hline & & $(1)^{\dagger}$ & $(2)^{\dagger}$ & $(3)^{\dagger}$ & Intens. \\
\hline 3726 & [O II] & 38.5 & 34.4 & $51^{*}$ & 36.0 \\
\hline 3729 & [O II $]$ & 28.5 & 24.4 & & 26.0 \\
\hline 3869 & [Ne III] & 67.4 & 67.2 & 53 & 67.3 \\
\hline 4102 & $\mathrm{H} \delta$ & 25.9 & 25.4 & 26.9 & 25.8 \\
\hline 4267 & $\mathrm{C}_{\text {II }}$ & 2.88 & 2.55 & 2.24 & 2.55 \\
\hline 4340 & $\mathrm{H} \gamma$ & 46.6 & 45.3 & 46.7 & 46.5 \\
\hline 4363 & [O III] & 2.65 & 2.48 & 2.7 & 2.55 \\
\hline 4471 & $\mathrm{He} \mathrm{I}$ & 7.03 & 7.28 & 8.0 & 7.3 \\
\hline 4686 & He II & 11.2 & 13.5 & 11.7 & 12.5 \\
\hline 4711 & [Ar IV] & 2.07 & 1.81 & 2.7 & 2.0 \\
\hline 4724 & {$[\mathrm{Ne}$ IV] } & & 0.030 & & 0.030 \\
\hline 4725 & [Ne IV] & & 0.035 & & 0.035 \\
\hline 4740 & [Ar IV] & 1.22 & 1.38 & 1.7 & 1.38 \\
\hline 4861 & $\mathrm{H} \beta$ & 100 & 100 & 100 & 100 \\
\hline 5007 & [O III] & 459 & 515 & 547 & 510 \\
\hline 5518 & [Cl III] & 0.42 & 0.648 & 1.33 & 0.65 \\
\hline 5538 & [Cl III] & 0.38 & 0.617 & 0.90 & 0.61 \\
\hline 5755 & [N II] & 2.26 & 3.08 & 2.76 & 2.7 \\
\hline 5876 & $\mathrm{He} \mathrm{I}$ & 16.9 & 23.8 & 22.6 & 22.6 \\
\hline 6312 & [S III] & 1.02 & 1.44 & 1.39 & 1.42 \\
\hline 6563 & $\mathrm{H} \alpha$ & 285 & 323 & 283 & 290 \\
\hline 6584 & {$[\mathrm{~N} \mathrm{II}]$} & 214 & 276 & 267 & 250 \\
\hline 6717 & [S II] & 16.0 & 17.0 & 13.2 & 16.0 \\
\hline 6731 & [S II] & 18.9 & 20.7 & 18.0 & 20.0 \\
\hline 7135 & [Ar III] & 21.9 & 18.4 & 18.6 & 18.4 \\
\hline 7320 & [O II] & & 2.37 & & 2.37 \\
\hline 7330 & [O II $]$ & & 2.04 & & 2.04 \\
\hline
\end{tabular}

$\dagger$ (1) Exter et al. (2004); (2) Liu et al. (2001); (3) Aller \& Keyes (1979). ${ }^{*}$ This is a blend of $\lambda 3726$ and $\lambda 3729$.

Liu et al. (2001). They are shown in Table 3. Considering the bad quality of the spectrum, the difference between our measurements and those of Liu et al. (2001) probably reflect the uncertainty in the intensities.

The extinction correction was made by assuming a theoretical ratio for the He II line ratio $\lambda 1640 / \lambda 4686 \AA$ at $T=10000 \mathrm{~K}$ and an $N_{\mathrm{e}}$ of $10^{3} \mathrm{~cm}^{-3}$. This leads to a value of the $\lambda 1640 \AA$ line as given in Col. (2) of Table 4. Together with the measured intensity of the line and the assumption of a normal extinction law (e.g. that of Fluks et al. 1994) leads to a value of the extinction $\mathrm{C}$ which is $50 \%$ higher than that given above. This was also noticed by Liu et al. (2001) who ascribed this to an abnormal extinction law in this direction. While this could be true there is no other evidence for it. The higher intensity of the $\lambda 1640 \AA$ line could be due to the poor measurement. Below it is shown that the $\lambda 1663 \AA$ line of O III] leads to an improbably high electron temperature. The conclusion, which we share with Liu et al. (2001), is that this line is probably spurious and should be ignored. This is indicative of the uncertainties in the UV intensities. If the extinction correction is made relative to $\lambda 1640 \AA$ using $E_{B-V}=0.43$, and the reddening curve of Fluks et al.(1994) we obtain the results shown in the last column of Table 4. Liu et al. (2001) derive the intensities using an extinction correction which is the same as ours in the visible and near UV but increases more steeply toward the $\lambda 1640 \AA$ line. As a consequence the C III] line at $\lambda 1909 \AA$ has an intensity only half as great as we have found. This increases the uncertainty of the carbon abundance by a factor of two.
Table 4. IUE spectrum of M 1-42.

\begin{tabular}{|c|c|c|c|c|}
\hline \multirow{2}{*}{$\begin{array}{c}\lambda \\
(\AA)\end{array}$} & \multirow[t]{2}{*}{ Ion } & \multicolumn{3}{|c|}{ Intensities } \\
\hline & & $(1)^{\dagger}$ & $(2)^{\sharp}$ & $(3)(\mathrm{I} / \mathrm{H} \beta)^{\star}$ \\
\hline 1640 & He II & 4.91 & 8.1 & 81 \\
\hline 1663 & O III] & 2.89 & 4.7 & 47 \\
\hline 1863 & $\mathrm{Al} \mathrm{III]}$ & 1.71 & 2.8 & 28 \\
\hline 1909 & C III] & 2.77 & 4.56 & 45.6 \\
\hline
\end{tabular}

$\dagger$ Measured intensity from low resolution spectra in units of $10^{-14} \mathrm{erg} \mathrm{cm}^{-2} \mathrm{~s}^{-1}$.

\# Intensity corrected for extinction in units of $10^{-12} \mathrm{erg} \mathrm{cm}^{-2} \mathrm{~s}^{-1}$.

${ }^{\star}$ Normalized to $\mathrm{H} \beta=100$.

Table 5. Electron density indicators in M 1-42.

\begin{tabular}{lcccc}
\hline \hline Ion & $\begin{array}{c}\text { Ioniz. } \\
\text { Pot. }(\mathrm{eV})\end{array}$ & $\begin{array}{c}\text { Lines } \\
\text { Used }\end{array}$ & $\begin{array}{c}\text { Observed } \\
\text { Ratio }\end{array}$ & $\begin{array}{c}N_{\mathrm{e}} \\
\left(\mathrm{cm}^{-3}\right)\end{array}$ \\
\hline$[\mathrm{S} \mathrm{II}]$ & 10.4 & $6731 / 6716$ & 1.25 & 1200 \\
{$[\mathrm{O}$ II $]$} & 13.6 & $3626 / 3729$ & 1.44 & 1600 \\
{$[\mathrm{~S} \mathrm{III}]$} & 23.3 & $33.5 / 18.7$ & 0.65 & 1400 \\
{$[\mathrm{Cl}$ III $]$} & 23.8 & $5538 / 5518$ & 0.924 & 1400 \\
{$[\mathrm{O}$ III $]$} & 35.1 & $51.9 / 88.4$ & 2.13 & 800 \\
\hline
\end{tabular}

\section{Chemical composition of the nebulae}

The method of analysis is the same as used in the papers cited in the introduction. First the electron density $N_{\mathrm{e}}$ and temperature $T_{\mathrm{e}}$ as function of the ionization potential are determined. Then the ionic abundances are determined, using density and temperature appropriate for the ion under consideration. Then the element abundances are found for those elements in which a sufficient number of ionic abundances have been derived.

\subsection{Electron density and distance}

The ions used to determine $N_{\mathrm{e}}$ are listed in the first column of Table 5. The ionization potential required to reach that ionization stage, and the wavelengths of the lines used, are given in Cols. 2 and 3 of the table. Note that the wavelength units are $\AA$ when 4 ciphers are given and microns when 3 ciphers are shown. The observed ratio of the lines is given in the fourth column; the corresponding $N_{\mathrm{e}}$ is given in the fifth column. The temperature used is discussed in the following section, but is unimportant since these line ratios are essentially determined by the density.

There is no indication that the electron density varies with ionization potential in a systematic way. As already pointed out by Liu et al. (2001) the [O III] lines always give a lower density than the other lines. Ignoring these lines, the electron density is $1400 \mathrm{~cm}^{-3}$. The error is about $20 \%$. It is interesting to compare this value of the density with the rms density found from the $\mathrm{H} \beta$ line. This depends on the distance of the nebula which isn't accurately known, and is estimated in the following paragraph as $5 \mathrm{kpc}$. For this calculation we shall use a diameter of $10^{\prime \prime}$. Both of these quantities are uncertain. The $\mathrm{H} \beta$ flux has been given above and the electron temperature will be discussed below. We obtain the uncertain rms values of $1200 \mathrm{~cm}^{-3}$. Since both methods are in fair agreement we will use their average, giving a density of $1300 \mathrm{~cm}^{-3}$ in the further discussion of the abundances. Notice that the densities determined from the three optical line ratios are the same as that determined from the infrared line ratios.

The distance to the nebula is estimated in the following way. The position of the nebula is within a few degrees of the direction 
Table 6. Electron temperature indicators in M 1-42.

\begin{tabular}{lcccc}
\hline \hline Ion & $\begin{array}{c}\text { Ioniz. } \\
\text { Pot. }(\mathrm{eV})\end{array}$ & $\begin{array}{c}\text { Lines } \\
\text { Used }\end{array}$ & $\begin{array}{c}\text { Observed } \\
\text { Ratio }\end{array}$ & $\begin{array}{c}T_{\mathrm{e}} \\
(\mathrm{K})\end{array}$ \\
\hline$[\mathrm{N}$ II $]$ & 14.5 & $5755 / 6584$ & 0.0108 & 8400 \\
{$[\mathrm{~S} \mathrm{III}]$} & 23.3 & $6312 / 18.7$ & 0.0079 & 7500 \\
{$[\mathrm{Ar}$ III $]$} & 27.6 & $7136 / 21.8$ & 4.39 & 6500 \\
{$[\mathrm{O}$ III $]$} & 35.1 & $4363 / 5007$ & 0.0050 & 9300 \\
{$[\mathrm{O}$ III $]$} & 35.1 & $5007 / 51.8$ & 2.83 & 7800 \\
{$[\mathrm{Ne}$ III] } & 41.0 & $3869 / 15.5$ & 0.136 & 7000 \\
\hline
\end{tabular}

of the galactic center. Coupled with its high radial velocity it is almost certain that it belongs to the group of galactic bulge planetary nebulae. It is likely somewhat closer to us than the galactic center for two reasons. First its diameter is larger than most galactic bulge PNe even though it has a normal density. Second and perhaps more important, the extinction to the nebula is not so large (see Sect. 3.3). Most galactic bulge PN have higher extinction. We conclude that the PN belongs to the galactic bulge but is closer to us than many of the galactic bulge nebulae. The actual value used $(5 \mathrm{kpc})$ only means that the distance probably lies between 4 and $6 \mathrm{kpc}$ and it might be even somewhat further away if it is in a region of reduced extinction. Note that the statistical distances given by various authors (as listed by Acker et al. 1992) are somewhat smaller, ranging from 2.6 to $4.2 \mathrm{kpc}$ and are also uncertain.

\subsection{Electron temperature}

A number of ions have lines originating from energy levels far enough apart that their ratio is sensitive to the electron temperature. These are listed in Table 6 which is arranged similarly to the previous table. The electron temperature remains constant as a function of ionization potential at a value of about $8000 \pm 1000$.

\subsection{Ionic and element abundances}

The ionic abundances have been determined using the following equation:

$\frac{N_{\text {ion }}}{N_{\mathrm{p}}}=\frac{I_{\text {ion }}}{I_{\mathrm{H}_{\beta}}} N_{\mathrm{e}} \frac{\lambda_{\mathrm{ul}}}{\lambda_{\mathrm{H}_{\beta}}} \frac{\alpha_{\mathrm{H}_{\beta}}}{A_{\mathrm{ul}}}\left(\frac{N_{\mathrm{u}}}{N_{\text {ion }}}\right)^{-1}$

where $I_{\mathrm{ion}} / I_{\mathrm{H}_{\beta}}$ is the measured intensity of the ionic line compared to $\mathrm{H} \beta, N_{\mathrm{p}}$ is the density of ionized hydrogen, $\lambda_{\mathrm{ul}}$ is the wavelength of this line, $\lambda_{\mathrm{H}_{\beta}}$ is the wavelength of $\mathrm{H} \beta, \alpha_{\mathrm{H}_{\beta}}$ is the effective recombination coefficient for $\mathrm{H} \beta, A_{\mathrm{ul}}$ is the Einstein spontaneous transition rate for the line, and $N_{\mathrm{u}} / N_{\text {ion }}$ is the ratio of the population of the level from which the line originates to the total population of the ion. This ratio has been determined using a five level atom.

The results are given in Table 7, where the first column lists the ion concerned, and the second column the line used for the abundance determination. The third column gives the intensity of the line used relative to $\mathrm{H} \beta=100$. The fourth column gives the ionic abundances assuming $T_{\mathrm{e}}=8000 \mathrm{~K}$, and the fifth column gives the Ionization Correction Factor (ICF). For the first eight elements in the table we judge that all the important ionization stages are observed so that the ICF is unity (or almost unity). For both $\mathrm{Fe}$ and $\mathrm{Si}$ a correction must be made for higher ionization stages than have been observed. We have done this by comparing the distribution of ionization stages of oxygen, neon and sulfur with what might be expected for Fe and Si. These ionization correction factors are quite uncertain. The element
Table 7. Ionic concentrations and chemical abundances in M 1-42. Wavelength in Angstrom for all values of $\lambda$ above 1000, otherwise in $\mu \mathrm{m}$.

\begin{tabular}{lccccc}
\hline \hline Ion & $\lambda$ & $\mathrm{I} / \mathrm{H} \beta^{\dagger}$ & $N_{\text {ion }} / N_{\mathrm{p}}$ & ICF & $N_{\text {el. } /} N_{\mathrm{p}}$ \\
\hline $\mathrm{He}^{+}$ & 5875 & 22.6 & 0.151 & & \\
$\mathrm{He}^{++}$ & 4686 & 12.5 & 0.010 & 1 & 0.161 \\
$\mathrm{C}^{++}$ & 1909 & 45.6 & $8.8(-4)$ & 1.2 & $10.5(-4)$ \\
$\mathrm{N}^{+}$ & 6584 & 250 & $9.43(-5)$ & & \\
$\mathrm{N}^{+}$ & 122. & 5.9 & $1.5(-4)$ & & \\
$\mathrm{N}^{++}$ & 57 & 269 & $5.46(-4)$ & 1.1 & $7.5(-4)$ \\
$\mathrm{O}^{+}$ & 3727 & 36.0 & $8.5(-5)$ & & \\
$\mathrm{O}^{++}$ & 5007 & 510 & $4.42(-4)$ & & \\
$\mathrm{O}^{++}$ & 51.8 & 383 & $7.25(-4)$ & & \\
$\mathrm{O}^{++}$ & 88.3 & 180 & $9.5(-4)$ & & \\
$\mathrm{O}^{+3}$ & 25.8 & 91.5 & $2.4(-5)$ & 1.0 & $8.3(-4)$ \\
$\mathrm{Ne}^{+}$ & 12.8 & 43.5 & $8.0(-5)$ & & \\
$\mathrm{Ne}^{++}$ & 15.5 & 497 & $3.6(-4)$ & & \\
$\mathrm{Ne}^{++}$ & 36.0 & 42.8 & $3.6(-4)$ & & \\
$\mathrm{Ne}^{++}$ & 3869 & 68.0 & $1.93(-4)$ & & \\
$\mathrm{Ne}^{+4}$ & 24.3 & 0.396 & $4.3(-8)$ & 1 & $4.4(-4)$ \\
$\mathrm{S}^{+}$ & 6731 & 20.0 & $1.93(-6)$ & & \\
$\mathrm{S}^{++}$ & 18.7 & 184 & $1.92(-5)$ & & \\
$\mathrm{S}^{++}$ & 6312 & 1.44 & $9.53(-6)$ & & \\
$\mathrm{S}^{+3}$ & 10.5 & 321 & $9.3(-6)$ & 1.0 & $2.8(-5)$ \\
$\mathrm{Ar}^{++}$ & 21.8 & 4.19 & $7.1(-6)$ & & \\
$\mathrm{Ar}^{++}$ & 7135 & 18.4 & $3.14(-6)$ & & \\
$\mathrm{Ar}^{+3}$ & 4740 & 1.38 & $1.07(-6)$ & & \\
$\mathrm{Ar}^{+4}$ & 13.1 & 0.63 & $2.05(-8)$ & 1.1 & $9.0(-6)$ \\
$\mathrm{Cl}^{+}$ & 14.4 & 1.41 & $3.6(-7)$ & & \\
$\mathrm{Cl}^{++}$ & 5538 & 0.61 & $1.96(-7)$ & & \\
$\mathrm{Cl}^{+3}$ & 11.8 & 0.844 & $5.8(-8)$ & 1.0 & $6.1(-7)$ \\
$\mathrm{Fe}^{+}$ & 26.0 & 0.255 & $6.7(-8)$ & & \\
$\mathrm{Fe}^{++}$ & 22.9 & 0.376 & $1.44(-7)$ & $2:$ & $4.0(-7):$ \\
$\mathrm{Si}^{+}$ & 34.8 & 0.16 & $4.8(-6)$ & $2:$ & $9.6(-6):$ \\
$\mathrm{C}^{++}$ & 4267 & 2.5 & $1.9(-3)$ & & \\
\hline
\end{tabular}

$\dagger$ Intensities given with respect to $\mathrm{H} \beta=100$. : Indicates uncertain value.

abundances, given in the last column, are therefore probably well determined only for the first eight elements. The carbon recombination line abundance is given at the end of the table. These abundances will be discussed in the next sections.

\section{Comparison with other abundance determinations}

Table 8 shows a comparison of our abundances with the most important determinations in the past 20 years. There is marginal agreement, usually to within a factor of two or three. It is rather surprising that such differences exist, even for oxygen. We discuss below reasons for this, which differ for different elements. A comparison is also made in the table with the solar abundance (Asplund et al. 2005). Note that for sulfur and chlorine more weight has been given to the abundance determination in meteorites since this determination is more accurate than for the sun itself. Neon and argon abundances are taken from the references given in Pottasch \& Bernard-Salas (2006).

The helium abundance has been derived using the results of Benjamin et al. (1999). For recombination of singly ionized helium, most weight is given to the $\lambda 5875 \AA$ line, because the theoretical determination of this line is the most reliable. 
Table 8. Comparison of abundances in M 1-42.

\begin{tabular}{lrrrrrr}
\hline \hline Elem. & Present & Liu & Exter & Aller & Solar & N6153 \\
\hline $\mathrm{He}$ & 0.161 & 0.148 & 0.151 & 0.17 & 0.098 & 0.14 \\
$\mathrm{C}(-4)$ & 10.5 & 0.63 & & & 2.5 & 6.8 \\
$\mathrm{~N}(-4)$ & 7.5 & 4.8 & 3.2 & 8.2 & 0.84 & 4.8 \\
$\mathrm{O}(-4)$ & 8.3 & 4.3 & 3.0 & 4.7 & 4.6 & 8.3 \\
$\mathrm{Ne}(-4)$ & 4.4 & 1.3 & 1.2 & 1.35 & 1.2 & 3.1 \\
$\mathrm{~S}(-5)$ & 2.8 & 1.2 & 0.71 & 1.9 & 1.4 & 1.9 \\
$\mathrm{Ar}(-6)$ & 9.0 & 3.7 & 3.4 & 4.6 & 4.2 & 8.5 \\
$\mathrm{Cl}(-7)$ & 6.1 & 1.9 & & 7.1 & 3.2 & 5.6 \\
\hline
\end{tabular}

References: Liu: Liu et al. (2001); Exter: Exter et al. (2004); Aller: Aller \& Keyes (1979); Solar: Asplund et al. (2005); N6153: Pottasch et al. (2003).

\subsection{Errors}

Here we will discuss the reasons for the abundance differences between what we have obtained and what has been found by other authors. This is interesting because we have used essentially the same visual measurements as these groups, and the comparison provides some insight into the reasons for the differences. From this it can be seen that the errors occur not only as a result of measurement errors, but they occur in the interpretation of the measurements: in the electron temperature which is derived and in the corrections made for unseen stages of ionization. It is even possible that errors in collisional cross-sections play a role when different lines are used to analyse a given ion. The ultraviolet measurements are somewhat different but affect only the carbon abundance.

The error of measurement for the IRS intensities given in Table 1 is small, probably not more than 5\%. A correction for missing emission in the short wavelength band is probably of the same order. The uncertainty in the collisional strengths introduce an error of $15-20 \%$ so that the total error for the ions of neon, sulfur and argon determined here with the IRS measurements is less than $25 \%$. This will also be true for the total abundances of these elements because the ICF is about unity. The error for the nitrogen and oxygen abundance is somewhat higher because the visual measurements are less certain (compare the different intensities found by different authors shown in Table 3). In addition the temperature is more important for these lines and the total errors may be twice as large. The largest error is for the carbon abundance which has already been discussed.

Bearing this in mind, it can be seen in Table 8 that our results sometimes differ by a large factor with previous determinations. Here we discuss the reasons for this for individual elements.

The differences with Liu et al. (2001) arise from two factors. First of all these authors use a higher electron temperature, derived from the [O III] $\lambda 4363 / \lambda 5007$ line ratio. While we find the same temperature from this ratio, it is inconsistent with the lower temperature found from other line ratios (see Table 6) and with the [O III] $\lambda 5007 / \lambda 51.8$ line ratio temperature. Secondly these authors use suspiciously low line intensities in the SWS infrared region of the spectrum. These effects both work in the same direction to decrease the abundance. A case in point is the $\mathrm{Ne}^{++}$abundance for which we find an abundance of a factor of 3 higher from the $\lambda 15.5 \mu \mathrm{m}$ line. The abundance differences with Exter et al. (2004) arise from the same temperature difference plus the fact that they did not have the infrared spectrum at their disposal and have to use ICFs. Aller \& Keyes (1979) used a temperature more similar to the one we used and therefore the differences are not as marked.
The problem of the temperature is not yet completely solved. This nebula does not show a marked temperature gradient as has been found in some nebulae. It is possible that temperature fluctuations do exist in this (and possibly other) nebula. For this reason it is desireable to make use of the infrared spectrum whereever possible, since the abundances derived from it do not have an important temperature dependence. The temperature uncertainty has the largest effect for carbon for which we find a value which is an order of magnitude higher than what Liu et al. (2001) find. Interestingly, this carbon abundance is almost equal to that found for M 1-42 from the recombination line of carbon, which we discuss in Sect. 5.3.

\subsection{Recombination line abundances}

Recombination lines of oxygen, carbon, nitrogen, neon and magnesium have been measured in this nebula and in several other $\mathrm{PNe}$ in recent years. Abundances determinations using these recombination lines often give higher abundances of the above elements (with the exception of magnesium) than the determination using the collisional lines (e.g. see Liu et al. 2001). The ratio of these two determinations varies from one nebula to the other, from about 10 down to about 1 . The highest value is found in M 1-42. The reason for this is at present unknown. The only clues are that the recombination lines of oxygen seem to be formed at a position different from the collisional lines of oxygen and closer to the center of the nebula ${ }^{1}$.

Also they are likely formed in a region of considerably lower electron temperature since these regions do not produce collisional line emission. At the same time a lower temperature can explain the lower electron temperature found from the Balmer jump. It has been suggested that the recombination lines are produced in small inclusions of high density and low temperature (see the workshop website in footnote). The existence of such inclusions is a real possibility but still unproved. If such regions exist they could affect our results because the temperature, while being low, might still be high enough that collisionally excited infrared lines could be formed there. No evidence for this is seen in our measurements. The abundances of those ions which can be measured both from infrared lines and from optical lines are very similar. At the same time the densities found from infrared line ratios ([S III] and [O III]) are essentilly the same as the densities determined from optical line ratios and do not suggest that they are formed in high density regions (see Table 5). Thus it seems unlikely that a great deal of the infrared emission from these lines originates in high density inclusions.

A strong indication that the abundances we have found are representative of the majority of the material in the nebula is the fact that they are in agreement with abundances found in all other PNe we have studied, when the galactic gradient is taken into account (see Pottasch \& Bernard-Salas 2006) for oxygen, sulfur, neon and argon which are not formed in the course of evolution. This is unlikely to be a random occurence and thus lends support to the reality of the abundances.

\subsection{Recombination line abundances of carbon}

The $\mathrm{C}^{++}$population can be obtained from the recombination line $\lambda 4267 \AA$ as well as from the collisionally excited line at $\lambda 1909 \AA$. The advantage of the recombination line is that it

\footnotetext{
1 Barlow has given a summary of these measurements in a recent workshop held in Beijing; they may be found on the website http://ast.pku.edu.cn/ xs2007/
} 
is not sensitive to the electron temperature, and is in the visual spectrum as well. It has the disadvantage that it is quite faint and thus difficult to measure accurately. It has been used for at least 30 years, and has been found to sometimes give higher $\mathrm{C}^{++}$populations than the collisional line. The reason for this is not clear (e.g. see the discussion of Liu et al. 2000 and the discussion in the previous subsection). We have redetermined the $\mathrm{C}^{++}$abundance using this line and the result is shown in the last line of Table 7. This is essentially the same value as is given by Liu et al. (2001) using the recombination line. While it is still a factor of 2 higher than that determined from the $\lambda 1909 \AA$ line the errors are quite large. It is therefore possible that both determinations give a similar result. While this may be true of carbon, the recombination lines of oxygen, nitrogen and neon give a much higher abundance for these elements than we have found (Liu et al. 2001).

\subsection{Comparison with NGC 6153}

The abundances in NGC 6153 are shown in the last column of Table 8 . There is a remarkable similarity with those of M 1-42. Although the abundances in M 1-42 may on average be slightly higher, the uncertainty in the individual elements is at least $20 \%$ to $30 \%$ and considerably higher for carbon. There are more similarities. First, the Balmer jump temperature for both nebulae is extremely low, of the order of $4000 \mathrm{~K}$. Second, the abundances found from the recombination lines is in both nebulae an order of magnitude higher than that found from the lines excited by collisions. Third, the morphology of both nebulae is similar in that no structure is seen in either nebula, yet neither is clearly round or elliptical.

The electron temperatures of both nebulae are very similar. At first sight this is expected since the abundances are almost the same. But even the details of the various temperature determinations are similar. For example, the temperature derived from the [O III] $\lambda 4363 \AA / 5007 \AA$ ratio is higher than the average in both nebulae while that derived from the [O III $] \lambda 5007 \AA / 51.8 \mu \mathrm{m}$ ratio is lower than the average. Furthermore the temperatures derived from the $[\mathrm{Ar}$ III] and $[\mathrm{S} \mathrm{III]}$ line ratios are lower than the average in both nebulae. It seems to us that these similarities are real and indicate that the same physical situation exists in both nebulae, and that this physical situation is the cause of the various, at first sight anomolous, measurements found in these nebulae. These are thus clues as to the physical situation existing in these nebulae.

\section{The central star}

\subsection{Stellar temperature}

Enough information is available to compute both the Zanstra temperature and the Energy Balance temperature of the central star. The Zanstra temperature requires the knowledge of the stellar apparent magnitude, the extinction and the $\mathrm{H} \beta$ flux. The last two quantities have already been given in Sect. 3. The apparent magnitude is listed by Acker et al. (1992) as $V=17.4$. Assuming that the star radiates as a blackbody the hydrogen Zanstra temperature is $T_{\mathrm{z}}(\mathrm{H})=63000 \mathrm{~K}$ and the ionized helium Zanstra temperature is $81000 \mathrm{~K}$. The Energy Balance temperature requires the knowledge of the ratio of the "forbidden" line intensities to $\mathrm{H} \beta$. This value is found by summing the intensities given above, and is about 35 after making a correction for unmeasured lines using the table of PreiteMartinez \& Pottasch (1983). This is a rather uncertain number.
To convert this value to a stellar temperature, the formulation of Preite-Martinez \& Pottasch (1983) is used, assuming blackbody radiation from the central star. The value of Case II (the nebula is optically thin for radiation which will ionize hydrogen but optically thick for ionized helium radiation) for the energy balance temperature is $\left(T_{\mathrm{EB}}\right)=100000 \mathrm{~K}$. If a model atmosphere had been used instead of a blackbody, the energy balance temperature would be lower. The low value of the hydrogen Zanstra temperature $\left(T_{\mathrm{z}}(\mathrm{H})\right)$ may be due to the nebula being optically thin to radiation ionizing hydrogen. An average stellar temperature of about $85000 \mathrm{~K}$ is a reasonable first approximation. This is indicated by the rather good agreement of the various methods.

\subsection{Radius and luminosity}

The stellar radius and luminosity are dependent on the distance of the nebulae which is difficult to obtain accurately. In Sect. 4.1 we estimated that the nebula is on the near side of the galactic center and its distance hss an uncertain value of $d=5 \mathrm{kpc}$. As a galactic bulge nebula it could have a distance of $8 \mathrm{kpc}$. These distances, combined with the magnitude of the star, lead to the stellar radius of $0.171 R_{\odot}$ and $0.438 R_{\odot}$. Once the radius is known the gravity may be computed assuming that the star has a mass of $0.6 M_{\odot}$. The value is $g=5.6 \times 10^{5}$ and $2.2 \times 10^{5} \mathrm{~cm} \mathrm{~s}^{-2}$. Knowing the temperature and radius of the star the luminosity may be found: $L / L_{\odot}=1400$ at $5 \mathrm{kpc}$ and 3500 at the less likely distance of $8 \mathrm{kpc}$.

\section{Evolutionary considerations and conclusions}

The abundances in M 1-42 are, at first sight, rather high. This probably is related to the fact that it is in the galactic bulge. We can use the PN abundances and abundance gradient given by Pottasch and Bernard-Salas (2006) to estimate the abundance expected at $3 \mathrm{kpc}$ from the galactic center, which would be the position of M 1-42 if it were at a distance of $5 \mathrm{kpc}$ from the sun. Their gradients lead us to expect the following average abundances at this position: $\mathrm{O} / \mathrm{H}=12.6 \times 10^{-4}, \mathrm{Ne} / \mathrm{H}=3.2 \times 10^{-4}$, $\mathrm{S} / \mathrm{H}=2.1 \times 10^{-5}, \mathrm{Ar} / \mathrm{H}=8.0 \times 10^{-6}$. These abundances are all within $50 \%$ of the values found for M 1-42. From this we conclude that M 1-42 has the abundances expected from a PN at about this distance from the galactic center, although the oxygen abundance is somewhat low compared to neon, sulfur and argon. The helium abundance is high; this usually occurs in nebulae in which hot bottom burning has taken place. But most models do not predict values of $\mathrm{He} / \mathrm{H}$ higher than 0.15 at any stage of their development. The high nitrogen/oxygen ratio indicates that hot bottom burning has occurred even though the ratio may be slightly lower than unity. According to Karakas (2003) this will occur in stars of masses greater than about $5 M_{\odot}$. The same models show that the carbon abundance increases relative to oxygen above $2.5 M_{\odot}$; the carbon/oxygen ratio becomes greater than unity above $3 M_{\odot}$, reaches a maximum at $3.5 M_{\odot}$ and begins to decline at higher masses. Carbon is expected to be a factor of at least 2 lower than oxygen when nitrogen is as abundant as oxygen. Because the carbon abundance in M 1-42 is uncertain, we do not put much weight on this discrepancy. Thus it seems likely that M 1-42 originated with a mass of slightly more than $5 M_{\odot}$. If this is so the rather low luminosity of the central star of this nebula, although quite uncertain, appears at first sight to be inconsistent with this high mass. This is because calculations of the evolution of temperature and luminosity of stars of different initial masses (e.g. Blocker et al. 2000) lead to the conclusion that a star of initial mass of $5 M_{\odot}$ will evolve into a central star 
of core mass of $0.7 M_{\odot}$ with a luminosity of $\log L=4$. This luminosity is considerably higher than we have found. The only way to reconcile these seemingly discrepant results would be if the $5 M_{\odot}$ star evolved into a star of $0.5 M_{\odot}$ core mass, due perhaps to a higher mass loss after the nuclear processes have taken place.

Acknowledgements. This work is based on observations made with the Spitzer Space Telescope, which is operated by the Jet Propulsion Laboratory, California Institute of Technology under NASA contract 1407. Support for this work was provided by NASA through Contract Number 1257184 issued by JPL/Caltech.

\section{References}

Acker, A., Marcout, J., Ochsenbein, F., et al. 1992, Strasbourg-ESO catalogue Aller, L. H., \& Keyes, C. D. 1987, ApJS, 65, 405

Asplund, M., Grevesse, N., \& Sauval, A. J. 2005, ASP Conf. Ser., ed. Bash, \& Barnes

Benjamin, R. A., Skillman, E. D., \& Smits, D. P. 1999, ApJ, 514, 307

Bernard Salas, J., Pottasch, S. R., Beintema, D. A., \& Wesselius, P. R. 2001, A\&A, 367, 949

Bernard-Salas, J., Pottasch, S. R., Feibelman, W. A., \& Wesselius, P. R. 2002, A\&A, 387, 301

Blocker, T., Herwig, F., \& Driebe, T. 2000, Mem. S. A. It., 71, 711

Cahn, J. H., Kaler, J. B., \& Stanghellini, L. 1992, A\&AS, 94, 399

Clegg, P. E., Ade, P. A. R., Armand, C., et al. 1996, A\&A, 315, L38

Condon, J. J., \& Kaplan, D. L. 1998, ApJS, 117, 361

Davey, A. R., Storey, P. J., \& Kisielius, R. 2000, A\&AS, 142, 85

de Graauw, T., Whittet, D. C. B., Gerakines, P. A., et al. 1996, A\&A, 315, 345
Exter, K. M., Barlow, M. J., \& Walton, N. A. 2004, MNRAS, 349, 1291 Fluks, M. A., Plez, B., de Winter, D., et al. 1994, A\&AS, 105, 311

Houck, J. R., Appleton, P. N., Armus, L., et al. 2004, ApJS, 154, 18 Hummer, D. G., \& Storey, P. J. 1987, MNRAS, 224, 801

Karakas, A. I. 2003, Thesis Monash Univ.

Kerber, F., Mignani, R. P., Guglielmetti, F., et al. 2003, A\&A, 408, 1029 Kingsburgh, R. L., \& Barlow, M. J. 1994, MNRAS, 271, 257

Liu, X.-W., Storey, P. J., Barlow, M. J., et al. 2000, MNRAS, 312, 585

Liu, X.-W., Luo, S.-G., Barlow, M. J., et al. 2001, MNRAS, 327, 141

Pottasch, S. R. 1984, Planetary Nebulae (Dordrecht: Reidel Publ. Co.)

Pottasch, S. R., \& Acker, A. 1989, A\&A, 221, 123

Pottasch, S. R., \& Beintema, D. A. 1999, A\&A, 347, 974

Pottasch, S. R., \& Bernard-Salas, J. 2006, A\&A, 457, 189

Pottasch, S. R., Wesselius, P. R., Wu, C. C., et al. 1977, A\&A, 54, 435

Pottasch, S. R., Preite-Martinez, A., Olnon, F. M., et al. 1986, A\&A, 161, 363

Pottasch, S. R., Beintema, D. A., \& Feibelman, W. A. 2000, A\&A, 363, 767

Pottasch, S. R., Beintema, D. A., Bernard Salas, J., \& Feibelman, W. A. 2001, A\&A, 380, 684

Pottasch, S. R., Beintema, D. A., Bernard- Salas, J., et al. 2002, A\&A, 393, 285

Pottasch, S. R., Bernard-Salas, J., Beintema, D. A., \& Feibelman, W. A. 2003, A\&A, 409, 599

Pottasch, S. R., Bernard-Salas, J., Beintema, D. A., \& Feibelman, W. A. 2004, A\&A, 423, 593

Preite-Martinez, A., \& Pottasch, S. R. 1983, A\&A, 126, 31

Ratag, M. A., Pottasch, S. R., Dennefeld, M., et al. 1997, A\&AS, 126, 297

Stanghellini, L., Corradi, R. L. M., \& Schwarz, H. E. 1993, A\&A, 276, 463

Torres-Peimbert, S., Peimbert, M., \& Daltabuit, E. 1980, ApJ, 238, 133

Tsamis, Y. G., Barlow, M. J., Liu, X.-W., et al. 2003, MNRAS, 345, 186

Webster, B. L. 1983, PASP, 95, 610

Zijlstra, A. A., Pottasch, S. R., \& Bignell, C. 1989, A\&AS, 79, 329

Werner, M., Roellig, T. L., Low, F. J., et al. 2004, ApJS, 154, 1 